CAMHS or other psychological services, however, one-quarter (24\%) were discharged home without further follow-up, and $3 \%$ left before assessment.

Conclusion Over a ten-year period, rates of self-harm in all age groups (10-24) increased by $22 \%$, but the largest increase was in youth aged under $10-14 \quad(+82 \%)$. The increasing rates as well as increases in methods of self-harm associated with higher lethality underline the need for interventions to reduce risk of repeat self-harm and suicide among this population. Presentations to hospital provide an opportunity to provide appropriate referral and treatment options for those engaging in self-harm. Having access to child and adolescent psychiatry services in pediatric hospitals would allow appropriate consultation, but in the absence of such services, it is essential that pediatric staff are competent in providing a therapeutic assessment and aware of appropriate service to refer on to.

\section{GP157 QUALITY, TRUSTWORTHINESS, READABILITY, AND ACCURACY OF MEDICAL INFORMATION REGARDING COMMON PEDIATRIC EMERGENCY MEDICINE-RELATED COMPLAINTS ON THE WEB}

${ }^{1}$ Ava Rothrock* ${ }^{2}$ Valeria Chavez, ${ }^{3}$ Jake Romney, ${ }^{4}$ Shira Isaak, ${ }^{5}$ Maria Pagane 'Sarah Swetland, '6Steven Rothrock, ${ }^{6}$ Silvio Chavez. 'Oviedo High School, Oviedo, USA; ${ }^{2}$ Vanderbilt University, Nashville, USA; ${ }^{3}$ Lake Mary Preparatory School, Lake Mary, USA; ${ }^{4}$ Winter Park High School, Winter Park, USA; ${ }^{5}$ Columbia University, New York, USA; ${ }^{6}$ Orlando Health, Orlando, USA

\subsection{6/archdischild-2019-epa.221}

Study Objectives To analyze the adequacy of information on the internet directed at parents regarding common acute pediatric complaints.

Methods Authors searched three internet search engines for four common acute pediatric complaints (child + fever, vomiting, cough, abdomen pain) assessing the first 20 results for each query. Website readability was evaluated using a composite of the Flesch-Kincaid Grade Level, Gunning Fog Scale, Simple Measure of Gobbledygook Score and Coleman-Liau Index. Quality and trustworthiness were independently assessed by two reviewers using JAMA Benchmark Criteria and National Library of Medicine (NLM) Trustworthy scores. Accuracy was independently assessed by two board-certified physicians (emergency medicine, pediatric emergency medicine) who analyzed text with website and author identifiers deleted. Accuracy was calculated by dividing the number of correct by the total number of correct and incorrect medical statements. Disagreements regarding physician accuracy were settled by a third physician.

Accuracy was defined as $\geq 95 \%$ correct, readability as an $8^{\text {th }}$ grade or lower reading level, high quality as $\geq 3$ JAMA criteria, and trustworthiness as an NLM total score $\geq 3$ with all sub-scores $>0$.

Features of accurate and inaccurate websites were compared using chi-square analysis for categorical data, and Mann-Whitney $\mathrm{U}$ for continuous and ordinal data. Association between website accuracy and search rank order was measured using Spearman's correlation coefficient. Inter-rater reliability of website accuracy, JAMA criteria and NLM scores was measured using Cohen's kappa (k).

Results Ninety-six websites that were duplicates or directed at health professionals were excluded, leaving 144 evaluable websites. Of these, 60 (42\%) were readable, 49 (34\%) were certified as reliable by the Health on the Net (HON) Foundation, $38(26 \%)$ had high quality JAMA criteria $(\mathrm{k}=0.68)$, and $44(31 \%)$ had reliable NLM trustworthy scores $(\mathrm{k}=$ 0.66). Physicians graded 87 websites $(60 \%)$ as accurate $(\mathrm{k}=$ 0.94). Professional medical organizations (hospitals, academic societies, governments) more frequently published accurate websites compared to individuals and non-professional websites. $(74 \%$ vs. $46 \%, \mathrm{p}<0.01)$. There was no correlation between accuracy and search rank order (rho $=-0.05,-0.21$ to $0.12,95 \% \mathrm{CI})$. There was no association between accuracy and physician authorship, quality, trustworthiness, readability, article age or HON certification.

Conclusion Most studied websites had poor quality, readability, and trustworthiness. Many websites were also inaccurate. Because inadequate web-based information might adversely influence parents' medical decisions, measures should be taken to ensure information related to acute pediatric complaints is of high quality, readable, trustworthy, and accurate.

\section{GP158 THE USE OF NITROUS OXIDE IN TEMPLE STREET EMERGENCY DEPARTMENT}

Danielle McCollum*, Jim Hickson, Niamh Whyte, Patrick Fitzpatrick. Children's University Hospital, Temple Street, Dublin, Ireland

\subsection{6/archdischild-2019-epa.222}

Background A visit to the Emergency Department (ED) can be stressful and frightening for children and their parents. ${ }^{1}$ Children perceive many of the interventions performed in ED as threatening, and a negative experience can have an impact on any future visits to hospital. ${ }^{2 ;} 3$ Through our procedural sedation programme, we aim to minimise this distress, reduce pain and improve the success rate of a procedure whilst ensuring patient safety at all times.

Aims The aim of this study is to review the use of Nitrous Oxide for procedural sedation within the Emergency Department between March and October 2018, and to evaluate the adverse events and outcomes.

Methods When performing procedural sedation in ED, a standardised sedation record is completed by the trained staff member and uploaded to a computer database. These forms were reviewed and data was input into Microsoft Excel for interpretation and analysis.

Results Over the study period, procedural sedation was performed on 142 occasions. The mean age of the patients was 6.9 years old (11 months - 15 year), and 68\% were male. The overall mean duration of sedation was 9.3 minutes (3minutes - 40minutes). The most common indication for use of Nitrous Oxide was in the management of a fracture or laceration (93/142). Other indications included removal of foreign bodies, lumbar punctures and IV cannulation. No serious adverse events occurred in the study period. Less significant side effects occurred in $22 \%$ of cases. Vomiting was the most common (27/142) side effect and was more likely to occur in children under 10 years of age.

Conclusion Nitrous oxide is used for a wide variety of reasons in our department and is generally well tolerated. Procedural sedation can be beneficial to patients in reducing distress and avoiding a general anaesthetic with prolonged hospital stays. The implantation of a database allows for continuous audit of our practices within the department and comparison with national and international standards. 